\title{
Susceptibility Pattern of Fosfomycin Against Drug Resistant Bacteria Obtained from Non - Urinary Clinical Samples in a Tertiary Care Hospital, South India
}

\author{
Premalatha Ethirajulu ${ }^{1}$, Jeyakumari Duraipandian ${ }^{2 *}$, Kandasamy Sankararaman $^{1}$, Sony MaryPaul ${ }^{1}$ \\ Priestly Vivekkumar ${ }^{3}$ and Sukumar Rathnamgiri ${ }^{1}$
}

${ }^{1}$ Department of Microbiology, Tagore Medical College \& Hospital, Affiliated to Tamilnadu DR MGR Medical University, Chennai, India ${ }^{2}$ Department of Microbiology, Jawaharlal Institute of Postgraduate Medical Education and Research, Karaikal. Pondicherry India ${ }^{3}$ Department of Pharmacology, Tagore Medical College \& Hospital, Affiliated to Tamilnadu DR MGR Medical University, Chennai, India

\section{ABSTRACT}

Background: Multi drug resistance (MDR) in bacterial infections has been an ever growing problem worldwide. To combat this some of the old drugs like fosfomycin used in the past are revived. The aim of our study was to determine the susceptibility of fosfomycin against Methicillin sensitive and Resistant Staphylococcus aureus, ESBL producing Escherichia coli, Klebsiella species and Metallo Beta Lactamase producing Pseudomonas aeruginosa isolated from the specimens other than urine and to evaluate the agreement between the two methods, disk diffusion and agar dilution methods performed as per CLSI guidelines.

Methods: First isolate of each species per patient $(\mathrm{n}=250)$ were tested for susceptibility to fosfomycin concomitantly by the disk diffusion and agar dilution methods described by CLSI guidelines and comparison of the two methods were studied.

Result: Staphylococcus aureus and ESBL E. coli were showing 100\% susceptibility, Whereas ESBL producing Klebsiella species showed $88 \%$ susceptibility to fosfomycin $200 \mu \mathrm{g}$ /disc and $80 \%$ and $72 \%$ by agar dilution method as per CLSI and EUCAST criteria. For MBL producing Pseudomonas aeruginosa, $90 \%$ isolates were susceptible to fosfomycin $200 \mu \mathrm{g} /$ disc and in agar dilution $(60 \%)(\leq 32 \mu \mathrm{g} / \mathrm{ml}) \mathrm{were}$ susceptible as per EUCAST criteria. Disk diffusion method showed good agreement for S.aureus and E.coli whereas moderate agreement for Klebsiella species and very poor for Pseudomonas aeruginosa.

Conclusion: Fosfomycin can be considered as an alternate drug to treat infections with multi drug resistant bacteria, not only for the UTI but for systemic infections also. This is achievable with establishment of breakpoint values and zone diameter for all common isolates.

Keywords: Fosfomycin, MRSA, ESBL E.coli, ESBL Klebsiella, MBL Pseudomonas Aeruginosa, Disc - Diffusion, Agar Dilution.

\section{Introduction}

Multi drug resistance (MDR) in bacterial infections has been an ever growing problem worldwide and emergence of resistance to antimicrobial drugs have been increasing in the organisms like Staphylococcus aureus, Enterococcus, Enterobactericeae group of organisms, Pseudomonas aeruginosa and Acinetobacter baumannii. ${ }^{[1]}$ The isolates of MDR organisms reduce the number of active drugs used to treat these infections. [2],[3] Infections by gram negative bacteria is becoming an immense challenge due to incipient of drug resistance among these pathogens, render even the broad spectrum and newer antibiotics resistant. ${ }^{[1]}$ Extended spectrum of Beta lactamases (ESBL), Amp $\mathrm{C}$ beta lactamases, carbapenemases producing gram negative bacteria and MRSA, VRE among gram positive bacteria have emerged as significant therapeutic challenge. The organisms called as "ESKAPE" ie) Enterococcus faecium, S. aureus, Klebsiella pneumoniae, A. baumanii, P. aeruginosa and Enterobacter species are the greatest threat in the present scenario, as they easily evade from the action of antibiotics. ${ }^{[4]}$

Nonetheless, drugs with considerable antimicrobial activity are dearth of in clinical practice since one decade and escalating progression of drug resistance coupled with a diminished antibiotic pipeline has led some to claim that a post-antibiotic era is eminent. ${ }^{[5]}$ To battle this problem some of the old drugs used in the past like colistin, polymyxin, tenocillin for gram negative bacteria and Fosfomycin for both gram positive and gram negative bacteria is revived. Out of these fosfomycin is phosphonic acid compound discovered in 1969 in Spain. ${ }^{[6]}$ It inhibits cell wall synthesis by inactivating phosphophenol pyruvate transferase enzyme. It is a broad spectrum and bactericidal antibiotic showing $90 \%$ or greater susceptibility to ESBL producing Enterobacteriaceae, CPE and also to MDR P.aeruginosa, MRSA and VRE. ${ }^{[7],[8],[9]}$ A.baumanni is intrinsically resistant to fosfomycin. ${ }^{[8]}$ There are few studies showing the action of fosfomycin against these commonly 
encountered bacteria isolated from the specimens other than urine. ${ }^{[10]}$ Hence the aim of our study was to determine the susceptibility of fosfomycin against S.aureus (both MSSA \& MRSA), ESBL producing Escherichia coli, Klebsiella species and MBL producing Pseudomonas aeruginosa isolated from the specimens other than urine and to evaluate the agreement between the two methods, disk diffusion and agar dilution method performed as per CLSI guidelines. ${ }^{[11]}$

\section{Mateiral \& Methods}

Sample Collection and Identification: First isolates of each species from a patient were included and total isolates tested were $(\mathrm{n}=\mathbf{2 5 0})$, of which fifty isolates of each species included were Methicillin sensitive S.aureus (MSSA), Methicillin Resistant S.aureus (MRSA), ESBL producing E.coli \& Klebsiella species and Metallo beta lactamase producing (MBL) P.aeruginosa were tested. These bacterial species were isolated from various clinical specimens from 2015 to 2016 as follows. MSSA ( $n=50$ from Pus -40 , Blood -8 and Peritoneal fluid -2$)$, MRSA ( $n=50$ from Pus -43, Blood-6, Synovial fluid-1), ESBL E.coli $(n=50$ from Pus-28, Blood -18, CSF-1, Synovial fluid -1), ESBL Klebsiella species ( $\mathrm{n}=50$ from Pus-14, Blood -15,CSF-2, Asicitic fluid - 1, Sputum -18) and MBL Pseudomonas aeruginosa ( $n=50$ from Pus - 48 and Sputum - 2). Urinary isolates were not tested in our study. All the isolates were identified by conventional methods. MRSA was detected by using Cefoxitin 30mg disc, ESBL by double disc synergy test and MBL by Imipenem - EDTA disk method were carried out. ${ }^{[12][13]}$

Antimicrobial Testing: The antimicrobial susceptibility testing to fosfomycin was determined concomitantly by the disk diffusion and agar dilution methods described by CLSI guidelines. ${ }^{[11]}$ For disk diffusion method, Mueller hinton agar with fosfomycin disk $200 \mathrm{mg}$ containing $50 \mathrm{mg}$ of $\mathrm{G}_{6} \mathrm{PO}_{4}$ was used. For agar dilution method, Mueller Hinton agar (Himedia) supplemented with $25 \mathrm{mg} / \mathrm{ml}$ of Glucose - $6 \mathrm{PO}_{4}$ was used. The MIC is defined as the lowest concentration of drug that inhibits visible growth of the organism. Control strains were included viz; S.aureus ATCC 29213, E. coli ATCC 25922 and P.aeruginosa ATCC 27853. (Himedia) Interpretative criteria for disk diffusion was based on CLSI breakpoints given for urinary isolates of E. coli and Enterococcus faecalis, whereas for agar dilution method both CLSI and EUCAST criteria were followed. EUCAST criteria are given for Enterobactericeae, Pseudomonas and Staphylococcus species. (Table 1) ${ }^{[14]}$

Data Analysis: All the findings were recorded and a susceptibility pattern of the disk diffusion method was compared with agar dilution method as the reference method. The results were analysed statistically using SPSS version 20. Agreement and discrepancies between the evaluated and reference methods are classified as Very major errors (VME), Major errors (ME), and minor errors (Mi). Value for the kappa coefficient, which gives measure of the percentage of agreement between the categorical results of susceptibility testing methods, were interpreted according to the classification by Landis and Koch. (Table 2) ${ }^{[15]}$

\section{Results}

The susceptibility pattern of fosfomycin to the different isolates Viz: Staphylococcus aureus (both MSSA \& MRSA), ESBL producing Escherichia coli, Klebsiella species and MBL producing Pseudomonas aeruginosa are given in the (Table 3)

Susceptibility Pattern of Fosfomycin Against S.aureus: Both MSSA 50/50 (100\%) and MRSA 50/50 (100\%) were showing susceptibility to fosfomycin $200 \mu \mathrm{g} / \mathrm{disc}$ and by agar dilution method. As zone diameter breakpoint (mm) is not available for S.aureus, the results were interpreted as per CLSI criteria given for E.coli and Enterococcus fecalis. The MIC value was interpreted according to the EUCAST criteria. All the isolates were having MIC value $\leq 32 \mu \mathrm{g} /$ ml. (Table 3)

\section{Susceptibility Pattern for Gram Negative Bacteria}

ESBL producing E. coli: All the isolates 50/50 (100\%) were susceptible to both the fosfomycin $200 \mu \mathrm{g} / \mathrm{disc}$ and agar dilution method as per CLSI criteria (Table 3)

ESBL Producing Klebsiella Species: The isolates 44/50 (88\%) were susceptible to fosfomycin $200 \mu \mathrm{g} / \mathrm{disc}$ and in agar dilution, 40/50 (80\%) were sensitive with MIC value $\leq 64 \mu \mathrm{g} / \mathrm{ml}$ and 10/50 (20\%) were resistant $(>64 \mu \mathrm{g} / \mathrm{ml})$ as per CLSI guidelines and following EUCAST criteria 36/50 $(72 \%)$ isolates were susceptible and 14/50 (28\%) were resistant $(\geq 32 \mu \mathrm{g} / \mathrm{ml})$. (Table 3)

MBL Producing Pseudomonas Aeruginosa: The isolates $45 / 50(90 \%)$ were susceptible and $5 / 50(10 \%)$ resistant to fosfomycin $200 \mu \mathrm{g} /$ disc and in agar dilution 30/50 (60\%) $(\leq 32 \mu \mathrm{g} / \mathrm{ml})$ were susceptible and $20 / 50(40 \%)$ resistant as per EUCAST criteria. But according to CLSI guidelines given for E. coli, the susceptibility of $70 \%,(\leq 64 \mu \mathrm{g} / \mathrm{ml})$, $10 \%$ Intermediate $(128 \mu \mathrm{g} / \mathrm{ml})$ and resistant $20 \%(\geq 256)$ was observed.

Comparison of Susceptibility Tests: The comparison of disk diffusion with gold standard agar dilution method was carried out. The categorical agreement was 100\% for Staphylococcus aureus (MSSA \& MRSA) and ESBL 
producing E. coli. No errors were found. Whereas for ESBL producing Klebsiella, the VME of $40 \%$ and $39.2 \%$ according to CLSI and EUCAST criteria respectively was perceived. No major and minor errors were observed. The kappa value was 0.70 and 0.51 as per CLSI and EUCAST guidelines which indicate good and moderate agreement respectively. (Table 4 \& 5)
In case of MBL producing Pseudomonas aeruginosa, the very major error of $50 \%$ and $75 \%$ was observed following CLSI and EUCAST criteria correspondingly. No major error was found. Minor error (10\%) was observed only by CLSI criteria. The kappa value was 0.412 and 0.286 according to CLSI and EUCAST which indicates moderate and fair agreement respectively. (Table $4 \& 5$ )

Table 1: Interpretive criterion of fosfomycin recommended by CLSI ${ }^{[11]}$ \& EUCAST [14]

\begin{tabular}{|c|c|c|c|c|c|c|}
\hline \multirow{2}{*}{ Standard and Organism } & \multicolumn{3}{|c|}{ MIC ( $\mu \mathrm{g} / \mathrm{ml})$} & \multicolumn{3}{|c|}{$\begin{array}{l}\text { Zone diameter breakpoint }(\mathrm{mm}) \\
\text { for the following interpretive criteria }\end{array}$} \\
\hline & $\mathrm{S}$ & I & $\mathbf{R}$ & $S$ & I & $\mathbf{R}$ \\
\hline \multirow{2}{*}{$\begin{array}{l}\text { CLSI - (Urinary tract isolate only) } \\
\text { Escherichia coli } \\
\text { Enterococcus fecalis }\end{array}$} & $\leq 64$ & 128 & $\geq 256$ & $\geq 16$ & $13-15$ & $\leq 12$ \\
\hline & $\leq 64$ & 128 & $\geq 256$ & $\geq 16$ & $13-15$ & $\leq 12$ \\
\hline $\begin{array}{l}\text { EUCAST } \\
\text { Enterobacteriaceae i.v }{ }^{a}\end{array}$ & $\leq 32$ & - & $>32$ & $N A^{b}$ & NA & NA \\
\hline $\begin{array}{l}\text { Enterobacteriaceae } \\
\text { (Fosfomycin trometamol, } \\
\text { uncomplicated UTI only) }\end{array}$ & $\leq 32$ & - & $>32$ & NA & NA & NA \\
\hline Pseudomonas species i.v ${ }^{d}$ & $\leq 32$ & - & $>32$ & NA & NA & NA \\
\hline Staphylococcus species & $\leq 32$ & - & $>32$ & NA & NA & NA \\
\hline
\end{tabular}

a i.v., intravenous, ${ }^{\mathrm{b}} \mathrm{NA}$, not available, ${ }^{\mathrm{c}} \mathrm{UTI}$, urinary tract infection; ${ }^{\mathrm{d}}$ Intravenous fosfomycin may be used in combination with other antibiotics to treat P. aeruginosa infections. S - Sensitive, I - Intermediate \& R - Resistant

Table 2: Kappa coefficient - by Landis \& $\operatorname{Koch}^{[15]}$.

\begin{tabular}{|c|c|}
\hline Kappa value & Strength of agreement \\
\hline$<0.2$ & Poor \\
\hline$>0.2-<0.4$ & Fair \\
\hline$>0.4-<0.6$ & Moderate \\
\hline$>0.6-<0.8$ & Good \\
\hline$>0.8-<1$ & Very good \\
\hline
\end{tabular}

Table 3: Interpretation of Fosfomycin MIC by CLSI and EUCAST criteria

\begin{tabular}{|c|c|c|c|c|c|c|c|c|}
\hline \multirow[t]{2}{*}{ Organism Tested } & \multicolumn{3}{|c|}{ Disk Diffusion } & \multicolumn{3}{|c|}{ Agar dilution-CLSI } & \multicolumn{2}{|c|}{ Agar dilution - EUCAST } \\
\hline & S 16 & $I(13-15)$ & R 12 & 64 & 128 & 256 & 32 & $>32$ \\
\hline MRSA $(n=50)$ & $\begin{array}{c}50 / 50 \\
(100 \%)\end{array}$ & 0 & 0 & $\begin{array}{c}50 / 50 \\
(100 \%) \\
\end{array}$ & 0 & 0 & $\begin{array}{c}50 / 50 \\
(100 \%)\end{array}$ & 0 \\
\hline $\operatorname{MSSA}(n=50)$ & $\begin{array}{c}50 / 50 \\
(100 \%)\end{array}$ & 0 & 0 & $\begin{array}{c}50 / 50 \\
(100 \%)\end{array}$ & 0 & 0 & $\begin{array}{c}50 / 50 \\
(100 \%)\end{array}$ & 0 \\
\hline ESBL E.coli $(n=50)$ & $\begin{array}{c}50 / 50 \\
(100 \%)\end{array}$ & 0 & 0 & $\begin{array}{c}50 / 50 \\
(100 \%)\end{array}$ & 0 & 0 & $\begin{array}{c}50 / 50 \\
(100 \%)\end{array}$ & 0 \\
\hline ESBL Klebsiella $(n=50)$ & $\begin{array}{l}44 / 50 \\
(88 \%)\end{array}$ & 0 & $\begin{array}{c}6 / 50 \\
(12 \%)\end{array}$ & $\begin{array}{l}40 / 50 \\
(80 \%)\end{array}$ & 0 & $\begin{array}{l}10 / 50 \\
(20 \%)\end{array}$ & $\begin{array}{l}36 / 50 \\
(72 \%)\end{array}$ & $\begin{array}{l}14 / 50 \\
(28 \%)\end{array}$ \\
\hline $\begin{array}{l}\text { MBL } P \text {. aeruginosa } \\
(n=50)\end{array}$ & $\begin{array}{l}45 / 50 \\
(90 \%)\end{array}$ & 0 & $\begin{array}{l}5 / 50 \\
(10 \%)\end{array}$ & $\begin{array}{l}35 / 50 \\
(70 \%)\end{array}$ & $\begin{array}{l}5 / 50 \\
(10 \%)\end{array}$ & $\begin{array}{l}10 / 50 \\
(20 \%)\end{array}$ & $\begin{array}{l}30 / 50 \\
(60 \%)\end{array}$ & $\begin{array}{l}20 / 50 \\
(40 \%)\end{array}$ \\
\hline
\end{tabular}

Table 4: Correlation of Disk Diffusion method with reference Agar dilution method.

\begin{tabular}{|l|c|c|c|c|c|c|}
\hline \multirow{2}{*}{} & \multicolumn{3}{|c|}{ CLSI (\%) } & \multicolumn{3}{c|}{ EUCAST (\%) } \\
\cline { 2 - 6 } & $\mathbf{V M}^{\mathbf{a}}$ & $\mathbf{M}^{\mathbf{b}}$ & $\mathbf{M i}^{\mathbf{c}}$ & $\mathbf{V M}$ & $\mathbf{M}$ & $\mathbf{M i}$ \\
\hline ESBL- klebsiella spp & $4 / 10(40 \%)$ & 0 & 0 & $8 / 14(39.2 \%)$ & 0 & 0 \\
\hline MBL-Pseudomonas aeruginosa & $5 / 10$ & 0 & $5 / 50$ & $15 / 20$ & 0 \\
& $50 \%$ & & $10 \%$ & $75 \%$ & & 0 \\
\hline
\end{tabular}

a - very major error, b-Major error, c- Minor error 
Table 5: Kappa value - Comparison of Disk diffusion with agar dilution method.

\begin{tabular}{|c|c|c|c|c|}
\hline & Kappa value & Asymp Std error ${ }^{\mathrm{a}}$ & Approx $T^{\mathrm{b}}$ & Approx sig \\
\hline $\begin{array}{l}\text { Measure of Agreement (kappa) } \\
\text { ESBL Klebsiella species CLSI criteria }\end{array}$ & 0.706 & 0.135 & 5.222 & 0.000 \\
\hline EUCAST criteria & 0.519 & 0.137 & 4.187 & 0.000 \\
\hline $\begin{array}{l}\text { MBL - P.aeruginosa } \\
\text { CLSI criteria }\end{array}$ & 0.412 & 0.135 & 4.187 & 0.000 \\
\hline EUCAST criteria & 0.286 & 0.108 & 2.887 & 0.004 \\
\hline No of valid cases & 50 & 50 & 50 & 50 \\
\hline
\end{tabular}

${ }^{a}$, not assuming the null hypothesis; ${ }^{b}$ using the asymptotic standard error assuming the null hypothesis.

\section{Discussion}

One form of fosfomycin is Fosfomycin trometamol which is used in the treatment of uncomplicated urinary tract infections (UTI) as an oral single dose regimen. The other is an intravenous (IV) form of fosfomycin - Fosfomycin di sodium salt, has been used in some European countries and in Japan. Successful outcomes were reported in some studies by using fosfomycin intravenously for infections other than UTI, although fosfomycin has not been approved for conditions other than UTI. [16], [17], [18] Our study of susceptibility pattern of fosfomycin against drug resistant gram positive cocci like MRSA and gram negative bacilli like ESBL producing Escherichia coli and Klebsiella species, MBL Producers Pseudomonas aeruginosa correlates well with many studies.

All our isolates tested were from the specimens other than urine, mainly the pus, blood, body fluids, and sputum. MRSA is known for its tenacious problem in both hospital and community acquired infections. ${ }^{[19]}$ Fosfomycin was found to be effective in the treatment of experimental MRSA osteomyelitis in rats. ${ }^{[20]}$ Our study showed that all the isolates of MRSA tested were $100 \%$ susceptibility to fosfomycin both by disc diffusion and agar dilution methods as per CLSI and EUCAST criteria. This is in consistent with the study conducted by Falgas etal, where 129 of $130(99.2 \%)$ of MRSA isolates were susceptible to fosfomycin. ${ }^{[21]}$ Even study by Lu CL etal observed that MSSA with $100 \%$ susceptibility like our observation and MRSA $89 \%$ of susceptibility both by CLSI and EUCAST criteria. ${ }^{[22]}$ But it differs from the study by Oksuz etal reported high fosfomycin resistance $(58 \%)$ in isolates of a ST 239 - MRSA - III clone. ${ }^{[23]}$

The effect of fosfomycin against ESBL producing Enterobactericeae particularly E. coli and Klebsiella species were studied. In our study E. coli isolates were susceptible to fosfomycin $200 \mu \mathrm{g} / \mathrm{disc}(100 \%)$ and also by agar dilution methods as per CLSI and EUCAST criteria. Studies conducted by Adil karadag etal, ${ }^{[18]}$ Pullukcu $\mathrm{H}$ etal, ${ }^{[24]}$ Endimiani etal, ${ }^{[25]}$ Tharavichitkul etal ${ }^{[26]}$ showed
$97.5 \%, 96.5 \%, 99.4 \%$ and $97.3 \%$ respectively. All these studies support our findings and also fosfomycin has high susceptibility against carbapenem resistant E. coli (95.1\%). Hence fosfomycin can be considered as an alternate drug for ESBL and Carbapenemase producing E. coli. ${ }^{\text {27], [28] }}$

In our study susceptibility of klebsiella species - ESBL producer to fosfomycin is $88 \%$ by DD method and $80 \%$ \& $70 \%$ by AD method following CLSI and EUCAST criteria respectively. This is in par with the study observed by Tharavichitkul etal ${ }^{[26]}$ but the study carried out by Endimiani etal [25] was showing only $63.2 \%$ of susceptibility to carbapenemase (KPC) producing Klebsiella pneumoniae, which was not tested in our study.

Moreover, study by Chitra etal ${ }^{[27]}$ in which Klebsiella species were showing $64.2 \%$ susceptibility by applying CLSI breakpoints and only $36 \%$ by EUCAST break point criteria. Also they stated that klebsiella species isolated from blood and sterile body fluids showed increased resistance compared to urine isolates, while E. coli were unvaryingly susceptible in all isolates. This comparison could not observe in our study as we have done in isolates other than urine. Perdigao etal ${ }^{[29]}$ in his studies reported in the same way that similar isolates tested by $\mathrm{E}$ test showed $85 \%$ and $48 \%$ susceptibility by CLSI \& EUCAST respectively.

The susceptibility pattern of fosfomycin against Pseudomonas aeruginosa were $70 \%$ and $60 \%$ by AD method according to CLSI and EUCAST criteria respectively, whereas $90 \%$ by DD method by applying criteria given for E. coli of urinary isolates in CLSI. Most of the studies showed less susceptibility of fosfomycin to Pseudomonas aeruginosa.

The comparison of disc diffusion method with agar dilution was carried out. The categorical agreement between two methods was $100 \%$ for S.aureus (both MSSA \& MRSA) and ESBL producing E.coli but errors were observed in between these two methods for Klebsiella species and Pseudomonas aeruginosa. In Klebsiella species, the very 
major error was $40 \%$ and $39.2 \%$ by CLSI \& EUCAST guidelines respectively. The kappa value was 0.706 and 0.519 in accordance with CLSI and EUCAST guidelines which showed good to moderate agreement. Our findings are different from the study conducted by $\mathrm{M}$ de cueto etal ${ }^{[10]}$ compared the DD method with AD method in which VME $0.7 \%$, Major error $10.9 \%$ and Minor error $3.6 \%$ was observed and study by Perdigao - Neto etal ${ }^{[29]}$ perceived very major Error of $4 \%$ only, which shows less error rate when compared to our study. To consider a susceptibility test adequate, CLSI recommends that if it obtains $<10 \%$ Minor error, $<3 \%$ major error and $1.5 \%$ VME. ${ }^{[1]}$ Also in our study disk diffusion method reporting greater susceptibility to ESBL - Klebsiella species than agar dilution method whereas study by $\mathrm{M}$ de cueto etal ${ }^{[10]}$ reported greater resistance. As our study showed VME of $40 \%$ for Klebsiella species which concludes that disc diffusion is not satisfactory to study the susceptibility pattern. No breakpoints were given for klebsiella species in CLSI and EUCAST guidelines. The study was done by using the criteria given for E. coli from urinary isolates in CLSI and Enterobacteriaceae in EUCAST. In our study ESBL E. coli and MRSA had significantly lower fosfomycin MICs than klebsiella species which is in accord to previous studies. [28][30]

For Pseudomonas aeruginosa the VME was 50\% and 75\% as per CLSI and EUCAST criteria respectively and kappa coefficient was 0.412 and 0.286 as per CLSI \& EUCAST guidelines. Our study is in accordance with the study by Perdigao - Neto etal ${ }^{[29]}$ where VME was $80 \%$ and $100 \%$ following CLSI and EUCAST respectively. Hence disc diffusion is not adequate to test the susceptibility pattern.

\section{Conclusion}

The existing evidences and our study concluded that fosfomycin has a high level of antimicrobial activity against isolates with high level of resistance to antimicrobial drugs such as MRSA, ESBL producing E. coli, Klebsiella species and MBL producing Pseudomonas aeruginosa. Our study has limitations as we tested only for fifty isolates each and clinical outcome following treatment is also not known. The feasible method to know the susceptibility is Disk diffusion method which shows good agreement for S.aureus and E.coli whereas only moderate agreement for Klebsiella species and very poor for Pseudomonas aeruginosa. The error rates were also high for these two isolates. To consider a susceptibility test adequate CLSI recommends that if it obtains $<10 \%$ Minor error, $<3 \%$ major error and $1.5 \% \mathrm{VME}$. Therefore, need further studies to establish the breakpoint criteria and to know the clinical outcomes. Hence fosfomycin can be considered as an alternate drug for treatment of infections with multi drug resistant bacteria, not only for the UTI but for systemic infections also. This is achievable with the establishment of breakpoint values and zone diameter for all the common isolates.

\section{References}

1. Magiorakos A P, Srinivasan A, Carey RB, Carmeli, Y, Falagas, M.E.,Giske,C.G, etal. Multi drug resistant, extensively drug resistant bacteria: an international expert proposal for interim standard definitions for acquired resistance. Clin Microbiol Infect 2012; 18:268-81.

2. Gupta k. Emerging antibiotic resistance in urinary tract pathogens. Infect Dis Clin North Am 2003; 17:243-59.

3. Falgas ME, Polemis M, Alexious VG, Marini Mastrogiammaki A, Kremastinou j, Vatopoulos AC. Antimicrobial resistance of Escherichia coli urinary isolates from primary care patients in Greece. Med Sci Monit 2008; 14: 75-79.

4. Boucher HW, Talbot GH, Bradley JS, Edwards, J.E., Gilbert D, Rice L.B, etal. Bad bugs, no drugs: no ESKAPE! An update from the infectious diseases society of America. Clin Infect Dis 2009; 48:1-12.

5. Appelbaum PC. 2012 and beyond: potential for the start of a second pre-antibiotic era? J Antimicrob Chemother. 2012; 67:2062-68.

6. Raz R. Fosfomycin an old new antibiotic. Clin Microbiol Infect 2012; 18: 4-7.

7. Popovic M, Sreinort D, Pillai S, Joukhadar C. Fosfomycin: an old, new friend? Eur J Clin Microbiol Infect Dis 2010; 29:127-42.

8. Falgas ME, Kastoris AC, Karageorgopoulos DE, Rafailidis PI. Fosfomycin for the treatment of infections caused by multidrug non - fermenting gram negative bacilli. A systematic review of microbiological, animal and clinical studies. Int J Antimicrob Agents 2009; 34: 111- 20.

9. Falgas ME, Kastoris AC, Kapaskelis AM, Karageorgopoulos DE. Fosfomycin for the treatment of multidrug - resistant including extended spectrum beta - lactamase producing Enterobacteriaceae infections: a systematic review. Lancet Infect Dis 2010; 10: 43- 50.

10. De cueto M, Lopez L, Hernandez JR, Morillo C, Pascual A. In vitro activity of fosfomycin against Extended - Spectrum - $\beta$ - Lactamase- producing Escherichia coli and Klebsiella pneumoniae:comparison of susceptibility testing procedures. Antimicrob Agents Chemother 2006; 50:368 - 70.

11. Clinical and Laboratory Standards Institute. Performance Standards for Antimicrobial Susceptibility Testing 27th ed. CLSI supplement M100, Clinical and Laboratory Standards Institute, Wayne, PA. 2017.

12. Pitout JDP, Gregson DB, Poirel I, McClure JA, Le P, Chruch DL. Detection of Pseudomonas aeruginosa producing metallo - beta lactamases in a large central laboratory. J Clin Microbiol 2005; 43:3129-35. 
13. Helene G., Laurence D.R, Jean- Louis G, Vincent J, Jerome, R. Comparison of Nine Phenotypic Methods for Detection of Extended- Spectrum $\beta$ - Lactamase production by Enterobacteriaceae. J.Clin.Microbiol 2011; 49: 1048 -57.

14. European Committee on Antimicrobial Susceptibility Testing. 13 December 2009. European Committee on Antimicrobial Susceptibility Testing. http: //www.eucast. org. Accessed 20 June 2017.

15. Landis JR, Koch GG. The measurement of observer agreement for categorical data. Biometrics 1977; 33:159-74. http://dx.doi.org/10.2307/2529310

16. Falgas ME, Giannopoulou KP, Kakolakis GH, Rafailidis, P.I. Fosfomycin use beyond urinary tract and gastrointestinal infections. Clin Infect Dis 2008; 46:1069-77.

17. Yao JDC, Moellering Jr RC. Antibacterial agents In: Murray PR, Bacon EJ, Jorgensen JH etal. Eds., Manual of clinical microbiology. 9th ed. Washington. ASM Press. 2007.

18. Karadag A, Cayci YT, Bilgin k, Gunaydin M, Eroglu, C. In vitro efficacy of fosfomycin against clinical strains. JMID 2014; 4: 55-58.

19. Yu xH, Song XJ, Cai Y, Liang BB, Lin DF, Wang R. In vitro activity of two old antibiotics against clinical isolates of Methicillin-Resistant Staphylococcus aureus. J Antibiot 2010; 63: 497 -99.

20. Poeppl W, Tabudic S, Lingscheid T, Plasenzotti R, Kozakowski N, Georgopoulos A, etal. Efficacy of fosfomycin in experimental osteomyelitis due to methicillin resistant Staphylococcus aureus. Antimicrob Agents Chemother 2011; 55:931-33.

21. Falagas ME, Maraki S, Karageorgopoulos DE, Kastoris AC, kapaskelis A, Samonis G. Antimicrobial susceptibility of gram positive non-urinary isolates of fosfomycin. Int $\mathrm{J}$ Antimicrob Agents 2010; 35:497-99.

22. Lu CI, Ltu CY, Huang YT, Liao CH, Teng LJ, Turnidge JD, Hsuech PR. Antimicrobial susceptibilities of commonly encountered bacterial isolates of fosfomycin determined by agar dilution and disk diffusion methods. Antimicrob Agents
Chemother 2011; 55: 4295-301. http://dx.doi.org 10.1128/ AAC00349-11.

23. Oksuz L, Dupieux C, Tristan A, Bes M, Etienne J, Gurler N. The high diversity of MRSA clones detected in a university hospital in Istanbul. Int J med Sci 2013; 10: 1740-45.

24. Pullukcu H, Tasbakan M, Sipahi OR, Yamazhan T, Aydemir S, Ulusoy S. Fosfomycin in the treatment of extended spectrum beta lactamase producing Escherichiae coli related lowerurinary tract infections. Int J Antimicrob Agents 2007; 29: $62-65$.

25. Endimiani A, Patel G, Hujer KM, Swaminathan M, Perez F, Rice LB, etal. In vitro activity of fosfomycin against blakpccontaining klebsiella pneumonia isolates including those non- susceptible to tigecycline and or/colistin. Antimicrob Agents. Chemother 2010; 54:526- 29.

26. Tharavichitkul P, Khantawa B, Bousoung V, Boonchoo M. Activity of fosfomycin against extended - spectrum beta lactamase producing Klebsiella pneumoniae and Escherichia coli in Mahoney Nakom Chiang Mai Hospital. J Infect Dis Antimicrob Agents 2005; 22: 125-26.

27. Chitra C, Kumar DRN, Shakti L, Diana SK, Balaji V. Technical and interpretative issues of fosfomycin susceptibility testing. IJMM 2015; 33:611-12.

28. Kasse M, Szabados F, Anders A, Gaterman SG. Fosfomycin susceptibility in Carbapenem - Resistant Enterobacteriaceae from Germany. J Clin Microbiol 2014; 52:1893-97.

29. Perdigao - Neto LV, Oliveira MS, Rizek CF, Carrilho CM, Costa SF, Levin AS. Susceptibility of multiresitant gram negative bacteria to fosfomycin and performance of different susceptibility testing methods. Antimicrob Agents Chemother 2014; 58:1763-67.

30. Falagas ME, Marakis, Karageorgopoulos DE, Kastoris AC, Mavromanolakis E, Samonis G. Antimicrobial susceptibility of mluti- drug resistant (MDR) and extensively drug resistant (XDR) enterobactericeae isolates to fosfomycin. Int J Antimicrob Agents 2010; 35:240-43.

*Corresponding author:

Dr. D.Jeyakumari, Professor of Microbiology, JIPMER, Arasalar complex, Beach Road, Karaikal - 609602, Pondicherry UT, India

Phone: +91 9865587080

Email: karailabscuddalore@yahoo.co.in

Financial or other Competing Interests: None. 